\title{
WIRELESS INDOOR CHANNEL MODELING: STATISTICAL AGREEMENT OF RAY TRACING SIMULATIONS AND CHANNEL SOUNDING MEASUREMENTS
}

\author{
Gus German, Quentin Spencer,Lee Swindlehurst \\ Dept. Electrical \& Computer Engineering \\ Brigham Young University \\ Provo, Utah 84602
}

\author{
Reinaldo Valenzuela \\ Wirelesss Communications Research Dept. \\ Bell Labs, Lucent Technologies \\ Holmdel, NJ 07733
}

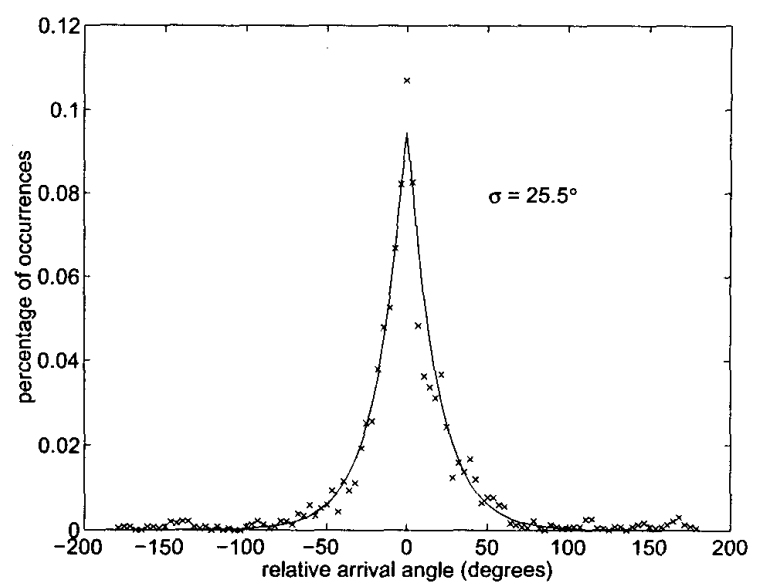

A statistical space-time model for indoor wireless propagation based on empirical measurements is compared with results from the deterministic ray-tracing simulation tool WiSE for the same environment. Excellent agreement is found in terms of the distributions of arrival times and angular spread for both modeling approaches. The WiSE package is also use to synthesize MIMO channel matrices and determine the theoretical capacity available in the tested environments. It is found that, for narrowband channels, the spatial clustering of the multipaths limits the capacity gains associated with increased array size.

\section{INTRODUCTION}

The parameters of any given wireless communications system are to a large extent determined by the propagation characteristics of the environment in which it is deployed. Numerous wireless channel models have been developed, primarily for propagation outdoors, in an effort to predict the effect of the channel without the expense of directly measuring it. Most of these models describe the temporal properties of the channel, but recent interest in the use of multiple antennas on both ends of the communication link have led to the development of models that also account for spatial spreading of the signal.

In this paper, we compare two space-time propagation modeling tools for indoor environments. The first is a statistical model developed in [1] using empirical measurements taken at $7 \mathrm{GHz}$. This model generalized the temporal-only model of [2] to include spatial multipath propagation statistics. A key component of both [1] and [2] is the grouping of multipath arrivals into clusters in both space and time. The second model we consider is one based on ray-tracing using the WiSE (for Wireless System Engineering) simulation tool developed by Lucent Technologies [3]. We used the WiSE package to simulate the same indoor propagation environment that was experimentally probed in [1], and compared the results with those presented in [1]. As described in the remainder of the paper,we found excellent agreement between the two models, from the heuristic clustering structure originally observed in the channel measurements to the measured statistical parameters in the model of [1]. Our observations complement those presented in [4], where agreement was found between results from the WiSE software and measurements taken in an outdoor urban environment.

With confidence that WiSE is accurately modeling our indoor channel, we used its output to synthesize the multiple-input

This work was supported by the National Science Foundation under Wireless Initiative Grant CCR 99-79452.
Fig. 1. Distribution of Relative Angles of Arrival for Measured Channel with Best Fit Laplacian Distribution.

multiple-output (MIMO) channel matrix that would result when employing antenna arrays at both transmitter and receiver. This allows us to quickly simulate various types of array sizes, geometries, and locations, and see their effect on capacity without a timeconsuming measurement campaign. We have found that, for the particular building environments tested, the effect of the multipath clustering is to limit the capacity gains that are predicted when the size of the array is increased, at least for narrowband channels.

\section{PROPAGATION MODELING}

In developing a propagation model for the wireless indoor channel, we may approach the problem from either a deterministic or a statistical viewpoint. A statistical model can be based on the bulk properties of channel sounding measurements which observe the channel directly. Alternatively, we may use classical propagation theory and a knowledge of the physical indoor environment to deterministically predict the behavior of the channel. Ideally, there should be a firm agreement between constructive, deterministic channel models and the statistical behavior observed in actual channels. 


\subsection{Statistical Channel Modeling}

As an example of a statistical model, Spencer, et al. [1] have observed that the indoor wireless channel follows the statistical clustering model proposed by Saleh and Valenzuela [2] and extended this model to include angle of arrival information in addition to the time domain impulse response. The time/angle impulse response is described in [1] by:

$$
h(t, \theta)=\sum_{l=0}^{\infty} \sum_{k=0}^{\infty} \beta_{k l} e^{j \phi_{k l}} \delta\left(t-T_{l}-\tau_{k l}\right) \delta\left(\theta-\Theta_{l}-\omega_{k l}\right)
$$

which groups arrivals into clusters (indicated by the index $l$ ) whose arrival is dictated by a Poisson process with rate parameter $\Lambda$. Ray arrivals within clusters (denoted by the index $k$ ) also arrive according to an independent Poisson process with rate parameter $\lambda$. As a result, we expect that the relative times of arrival for both clusters and individual rays will be distributed exponentially.

The additional angular dependence of the model describes clusters that are spatially localized around a mean cluster angle of arrival, $\Theta_{l}$. The parameter $\omega_{k l}$, which represents the deviation of each ray's angle of arrival from the mean cluster angle, is described by an independent Laplacian random process in which the distribution of $\omega_{k l}$ is given by:

$$
\mathbf{f}_{\omega_{k l}}(\omega)=\frac{1}{\sqrt{2 \sigma}} e^{-\left|\frac{\sqrt{2} \omega}{\sigma}\right|}
$$

By simultaneously measuring the channel impulse response in both time and arrival angle, it was observed in [1] tha the multipath arrivals accurately fit into the proposed clustering model Most significantly, the distribution of relative angles of individual arrivals closely followed the Laplacian distribution from the model, as seen in Figure 1. This plot was generated using data collected at $7 \mathrm{GHz}$ by taking the histogram of the relative angles of arrival over all of the measurements using 100 equally spaced bins between $-180^{\circ}$ and $180^{\circ}$. The solid line represents the Laplacian distribution that minimizes the squared error from samples of the data integrated over identical bin widths.

\subsection{Deterministic Channel Modeling}

As a complementary approach, ray-tracing and computational geometry can be used to predict the impulse response of the indoor wireless channel based on transmitter and receiver characteristics and the physical reflection/transmission environment of the building. Lucent Technologies has developed a 3-D predictive simulator for wireless channel propagation referred to as WiSE for Wireless System Engineering. It makes use of computational geometry, building environment descriptions, and a complex propagation model to predict the highest strength propagation paths from arbitrary transmitter and receiver locations within a static environment [3]. Three dimensional ray-tracing is used to include the effects of frequency, polarization, the dielectric properties of encountered materials, diffraction around corners, and antenna directivity. To speed up the computationally intensive task of predicting all possible illumination paths from transmitter to receiver, only rays with power above a fixed threshold are perpetuated through the model. Also, rays which are outside of the three dimensional cone illuminated by reflections off of a particular wall interface are discarded. This simulation tool allows deterministic prediction of the impulse response of wireless channels for both time and angle of arrival.

By comparing the channel impulse responses which result from both statistical observation and deterministic simulation, we hope to show consistency between the two modeling approaches and to

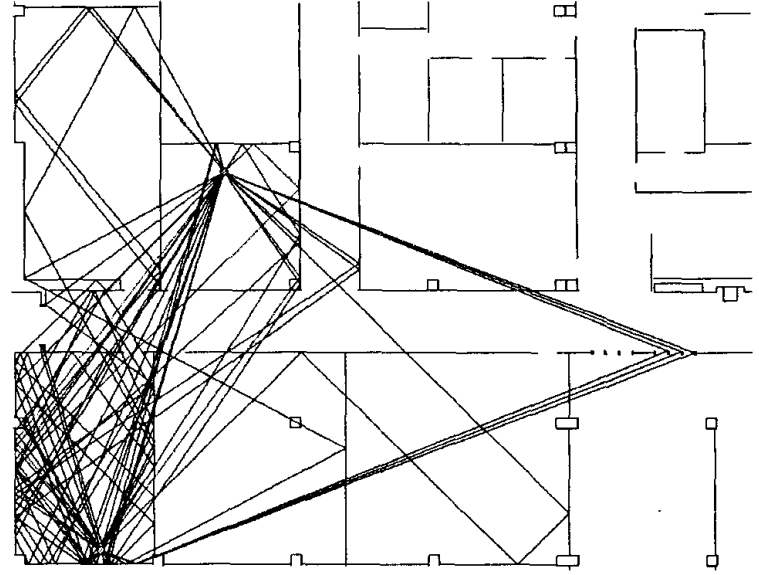

Fig. 2. Typical Results of Ray Tracing Simulation for a single transmitter/receiver location pair.

validate the major features of the results. Also, the ray-tracing simulation provides full definition of each propagation path. This allows some explanation of the underlying causes of the observed clustering structure in the channel. In particular, it is possible to measure the statistics of the angles of departure from the transmitter and to observe their possible correlation with the angles of arrival at the receiver without a complex system capable of directly measuring this information. This would provide a bridge between these two channel modeling paradigms and their contribution to the design of higher capacity indoor wireless networks and MIMO systems.

\section{METHODOLOGY}

We used the WiSE Simulation Package [3] to duplicate the $7 \mathrm{GHz}$ channel sounding experiments performed by Spencer [5] in the W.W. Clyde Engineering Building located on the Brigham Young University Campus in Provo, Utah. The Clyde Engineering building is representative of steel-reinforced, cement block construction. The parameters of the simulation were set to most closely match the operation of the original measurement system. An example of the ray-tracing results from these simulations is shown in Figure 2 . The apparent system power was normalized by adjusting the simulation ray power threshold to generate the same number of arrivals taken over all of the data cumulatively as were detected in the measurements. In grouping the impulse response data into clusters for analysis, we used the same algorithm that was applied to the earlier results.

The building geometry database needed for simulations was taken from original architectural CAD files. To reduce the complexity of the geometry, it was assumed that the dominant paths in the channel impulse response remained in the same horizontal plane as the transmitter and receiver. Initially, the building model of a single floor consisted of over 2500 wall entities with uniform dielectric composition. Using this as a starting point, we condensed the model to focus on the location and properties of scatterers in the building. This resulted in a final model with much lower wall entity density and correspondingly lower simulation time with improved statistical results. 


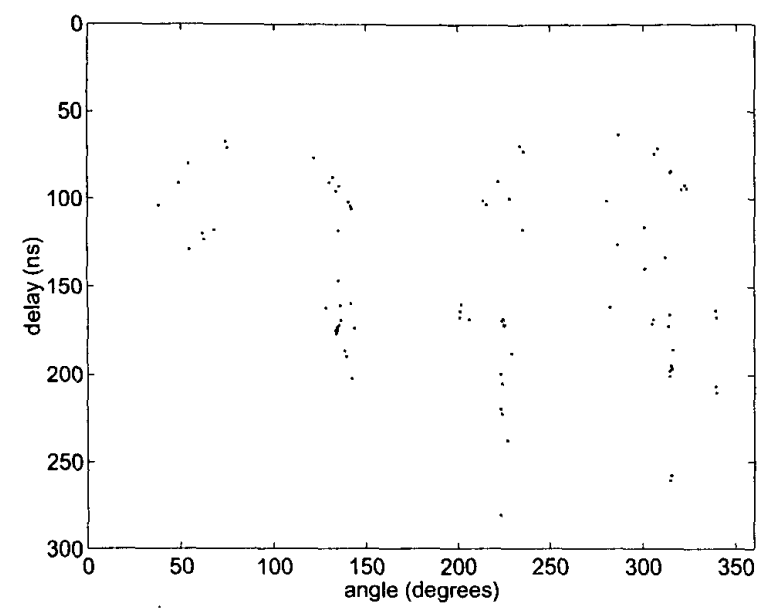

Fig. 3. Typical Arrival Angle vs. Time Scatter Plot for Simulated Indoor Channel.

\section{4: RESULTS}

The clustering:structure in both time and angle of arrivall observed in: [11]] was immediately; evident in the simulation results, as seen: in Figure 3. Using, the same methods: as before, the ray-tracing: data were: used! to estimate: the process parameters of the extended clustering model' of (1). To derive the cluster arrival rate parameter $\Lambda$, the first arrival in each cluster was: taken as the cluster arrival: time, and the relative delays were measured with respect to the arrival time of the previous cluster. This leaves out the first cluster for each data set, which is assumed in the clustering model to be equivalent to the bulk delay of the line-of-sight path. A histogram of the resulting relative cluster times was fit to an exponential PDF using least squares (as was done for all other curve fits presented here). The estimated parameter was $1 / \Lambda=18.94 \mathrm{~ns}$. Similarly, $\lambda$ was estimated by measuring the time of arrival for each arrival within a cluster with respect to the previous arrival, and curve fitting an exponential PDF to a histogram of the aggregated results for 50 total transmit-receive location pairs. In this case the estimated parameter was $1 / \lambda=5.97 \mathrm{~ns}$. Figures 4 and 5 illustrate the curve fitting used to estimate $\lambda$ and $\Lambda$, respectively. The second of these two plots actually shows the complimentary CDF rather than the PDF (although the curve fitting was to the PDF) because it is less noisy and better illustrates the quality of fit.

Finally, the parameter $\sigma$ representing the standard deviation of the angle of arrival with respect to the mean angle for each cluster was estimated similarly, using a histogram of all the aggregated clusters. This is illustrated in Figure 6. As in the previous empirical data, the fit to a Laplacian distribution was very good. The resulting standard deviation was $23.4^{\circ}$. A comparison of the statistical parameters estimated from the ray-tracing data with the original parameter estimates from empirical channel measurements in [1] is shown in Table 1. The comparison indicates surprisingly close agreement between the results.

With knowledge of both transmit and receive angles as well as the individual path gains, a MIMO channel transfer matrix can be synthesized from the ray-tracing data. For the narrowband case, where we assume that the impulse response associated with each path is a simple complex scalar, the expression for the channel

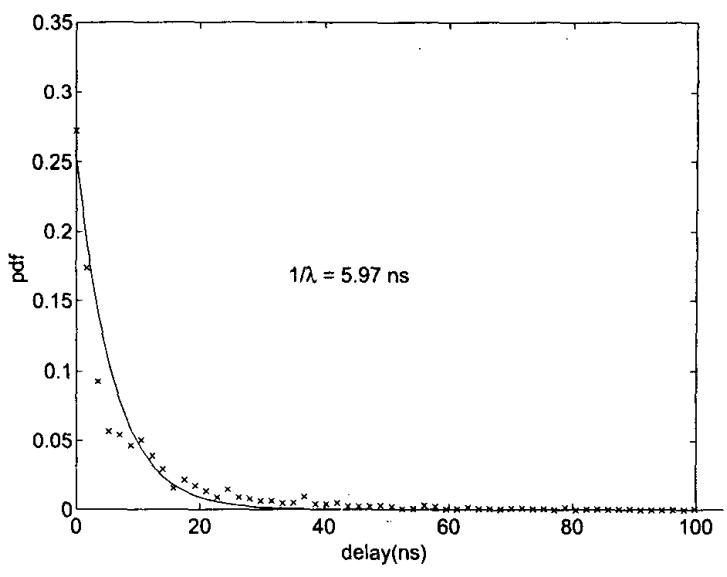

Fig. 4. Distribution of Time Intervals Between Ray Arrivals.

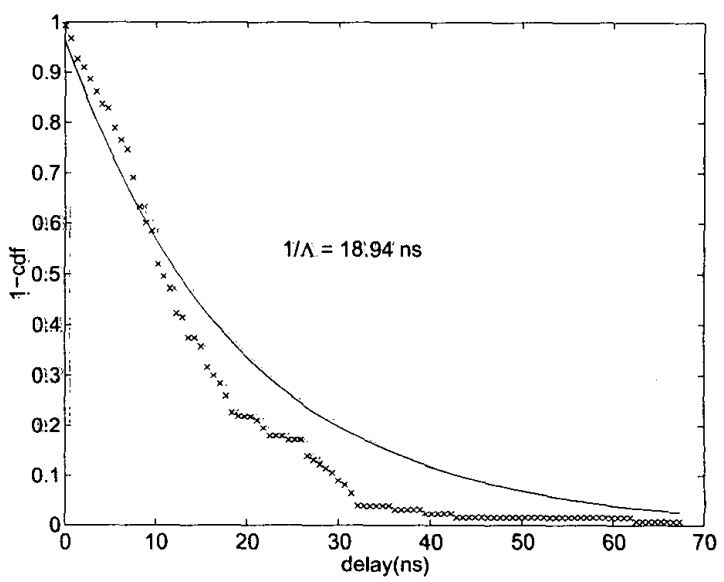

Fig. 5. Complementary Cumulative Distribution of Time Intervals Between Cluster Arrivals.

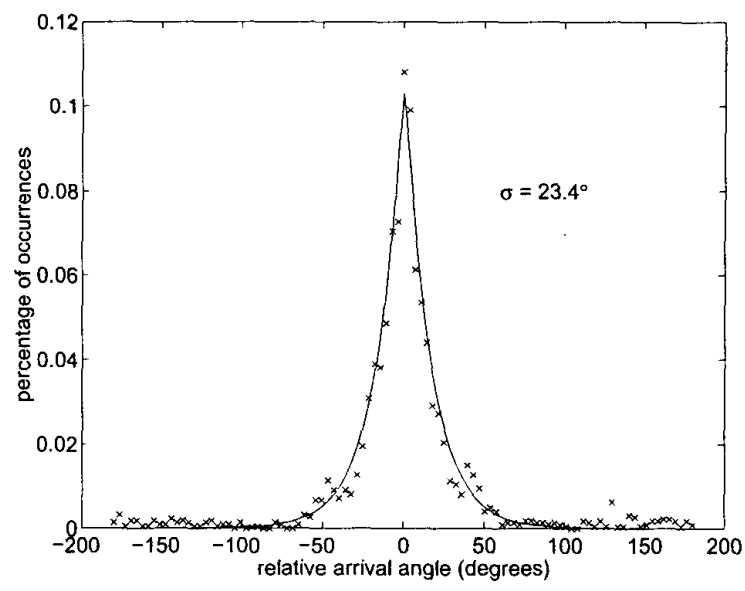

Fig. 6. Distribution of Relative Angles of Arrival for Simulated Channel with Best Fit Laplacian Distribution. 
matrix takes the following form:

$$
\mathbf{H}=\sum_{l=0}^{L} \boldsymbol{\alpha}_{T}^{T}\left(\theta_{T_{l}}\right) \beta_{l} \alpha_{R}\left(\theta_{R_{l}}\right)
$$

where $\beta_{l}$ is the individual complex path gain, $\theta_{T_{l}}$ and $\theta_{R_{l}}$ are the transmit and receive angles relative to some reference, and $\alpha_{T}(\theta)$ and $\alpha_{R}(\theta)$ are the respective steering vectors of the transmit and receive arrays for an arbitrary angle $\theta$. To study the effects of array configuration and signal to noise ratio (SNR) on capacity, we synthesized the channel for uniformly spaced linear transmit and receive arrays with element spacing of $\lambda / 2$ and uniformly random phase for each ray in the channel. Both the transmit and receive arrays were assumed to have the same number of elements. It was observed that, on average, the channel capacity was relatively insensitive to the orientation of the arrays, so we reduced the influence of orientation as a parameter by randomizing the array angle uniformly over 10 trials at each location. We also normalized each of the synthesized channel matrices to have unit Frobenius norm.

The capacity of the channel was then calculated over a range of SNR values using the water filling solution outlined, for example, in [6]. For each value of SNR and array size, we selected the value of capacity that was achieved or exceeded by $90 \%$ of the 500 simulated channel realizations. This represents the capacity that was achieved by the channel with a $10 \%$ probability of outage over all 50 transmit/receive locations with random array orientations. Figure 7 shows this outage capacity as a function of array size and system SNR. The capacity of this simulated indoor channel is insensitive to increases in the number of elements in a linear array except when operating at high SNR. This is mainly due to the fact that, in most of the data sets, a single dominant cluster of arrivals was present. The spatial diversity of the transmit and receive arrays will yield capacity improvements only when the SNR is high enough for clusters arriving from other directions to contribute power above the noise floor. At low SNR, the capacity of the channel does not increase linearly with the size of the arrays. As SNR increases, the capacity gain from increasing the array sizes is closer to linear.

\section{CONCLUSIONS}

Our results indicate that ray-tracing simulation of indoor spacetime propagation accurately reflects the general structure and statistics of data collected empirically. Consequently, ray-tracing can be considered reasonably reliable for generating data to characterize the temporal and spatial characteristics of indoor multipath channels, without the need for costly field measurements. Using the WiSE ray-tracing tool to simulate the propagation characteristics of a particular indoor environment on the BYU campus, we calculated the channel capacity that would result for a variety of array sizes, orientations, and SNRs. We found that, due to the temporal and spatial clustering of the multipath arrivals, increasing

\begin{tabular}{|c|c|c|}
\hline & Ray-Tracing & Empirical \\
\hline$\sigma$ & $23.4^{\circ}$ & $25.5^{\circ}$ \\
$1 / \Lambda$ & $18.94 \mathrm{~ns}$ & $16.8 \mathrm{~ns}$ \\
$1 / \lambda$ & $5.97 \mathrm{~ns}$ & $5.17 \mathrm{~ns}$ \\
\hline
\end{tabular}

Table 1. Comparison of estimated parameters from ray-tracing simulations and emipirical measurements.

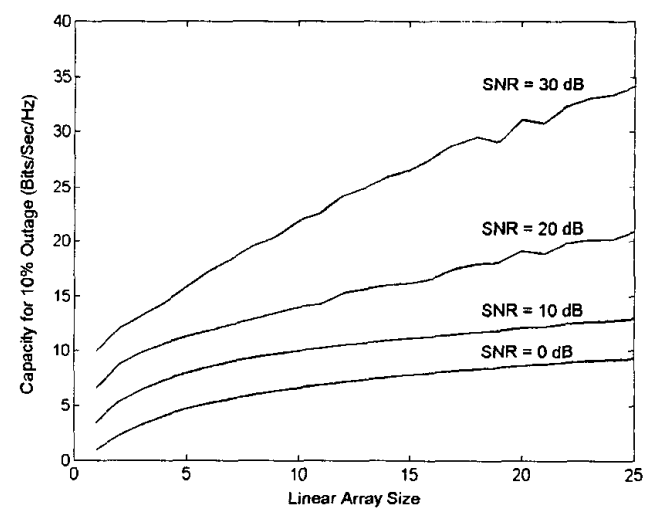

Fig. 7. Channel Capacity for $10 \%$ Outage as a function of Array Size and SNR.

array size does not necessarily result in a linear increase in channel capacity.

\section{REFERENCES}

[1] Q. H. Spencer, B. D. Jeffs, M. A. Jensen, and A. L. Swindlehurst. Modeling the statistical time and angle of arrival characteristics of an indoor multipath channel. IEEE Journal on Selected Areas In Communications, 18(3):347-360, March 2000.

[2] Adel A. M. Saleh and Reinaldo A. Valenzuela. A statistical model for indoor multipath propagation. IEEE Journal on Selected Areas of Communications, SAC-5:128-13, February 1987.

[3] Steven J. Fortune, David M. Gay,et al. Wise design of indoor wireless systems. IEEE Computational Science and Engineering,2 (1):58-68, Spring 1995.

[4] V. Erceg, S. Fortune, et al. Comparisons of a computer-based propagation prediction tools with experimental data collected in urban microcellular environments. IEEE Journal on Selected Areas in Communications, 15(4):677-684, May 1997.

[5] Quentin H. Spencer. Modeling the time and angle of arrival characteristics of an indoor multipath channel. Master's thesis, Brigham Young University,Dec ember 1996.

[6] Gregory G. Raleigh and John M. Cioffi. Spatio-temporal coding for wireless communication. IEEE Transactions on Communications, 46(3):357-366, March 1998. 